\title{
Vorhofflimmern - ein rhythmologisches Problem mit wachsender Bedeutung
}

${ }^{1}$ II. Medizinische Abteilung, Asklepios Klinik St. Georg, Hamburg.

\author{
Erik Wissner, Julian K.R. Chun, Karl-Heinz Kuck
}

Vorhofflimmern stellt eines der dringlichsten rhythmologischen Probleme unserer Gesellschaft dar, welches aufgrund der demographischen Entwicklung zukünftig an Bedeutung zunehmen wird. Dieser Herausforderung stellt sich in Deutschland das German Competence Network on Atrial Fibrillation (AFNET), einem Kompetenznetzwerk, das sich ausschließlich dem Thema Vorhofflimmern widmet. In dieser Ausgabe von Herz geben Breithardt et al. [1] einen Überblick über die gewonnenen Erkenntnisse sowohl aus dem deutschlandweiten Patientenregister als auch aus den vom AFNET konzipierten klinischen Studien.

Als Schwerpunkt in der Behandlung des $\mathrm{Pa}$ tienten mit Vorhofflimmern gilt die Thromboembolieprophylaxe, die trotz bestehender Leitlinien noch immer zu gering genutzt wird. Die richtliniengetreue Antikoagulation des Patienten mit Vorhofflimmern bedarf nicht nur der Behandlung durch den Kardiologen, sondern vielmehr des gesamten Spektrums und Zusammenspiels an Fachärzten. Eine Zusammenfassung dieses komplexen Themas gibt der Beitrag von Antz et al. [2], der u.a. auch die in den Leitlinien genutzten Empfehlungs- und Effizienzgrade beinhaltet.

Obgleich neue interventionelle Therapieformen zur Bekämpfung von Vorhofflimmern über die letzten Jahre erheblich an Bedeutung zugenommen haben, stellen die rhythmusstabilisierende und die frequenzkontrollierende Therapie weiterhin die primären Behandlungsformen dar. Somit ist der Weiterentwicklung auf diesem Gebiet die höchste Priorität zuzumessen. Hierzu berichten Schmitt et al. [3] von den Neuentwicklungen antiarrhythmischer Medikamente. Insbesondere werden das atrial-selektive Vernakalant und das dem Amiodaron verwandte Dronedaron besprochen.

Aufgrund demographischer Faktoren ist eine $\mathrm{Zu}-$ nahme der Prävalenz und Inzidenz herzinsuffizienter Patienten mit Vorhofflimmern zu beobachten. Den Herausforderungen und abweichenden Behandlungsmethoden des Vorhofflimmerns in diesem Patientenkollektiv widmet sich der Artikel von Straube et al. [4], der neben der medikamentösen Therapie auch invasive Strategien wie die biventrikuläre Schrittmacherimplantation mit konsekutiver His-Bündel-Ablation oder die Vorhofflimmerablation beleuchtet.

Eine bahnbrechende Beobachtung durch Haïssaguerre et al. [5], die den Pulmonalvenen erstmals eine führende Rolle in der Initiation des Vorhofflimmerns zusprach, führte zu einer neuen Ära in der interventionellen Elektrophysiologie. Ist die Pulmonalvenenisolation als Endpunkt heute meist fester Bestandteil der invasiven Therapie bei symptomatischem, antiarrhythmikarefraktärem Vorhofflimmern, befindet sich die Entwicklung der geeigneten Kathetertechnologie weiterhin in Bewegung. Zwar ist die Nutzung von Hochfrequenzstromenergie die dominante Energieform, doch sind gerade in den letzten Jahren neue Alternativen entwickelt worden. Hier sind besonders die kryo-thermalen Ballonverfahren und der hochfokussierte Ultraschall zu nennen. Der Artikel von Schmidt et al. [6] beschreibt diese neuen Technologien und gibt einen Überblick über den heutigen Forschungsstand.

Ein weiteres, hochaktuelles Thema in der invasiven Elektrophysiologie und Gegenstand des Artikels von Chun et al. [7] ist die Evaluation ferngesteuerter robotischer Ablationsverfahren. Diese werden in spezialisierten Zentren mit der Prämisse eingesetzt, gängige Ablationsprozeduren zu verkürzen, Durchleuchtungszeiten $\mathrm{zu}$ verringern und auch weniger erfahrenen Untersuchern einen schnelleren Einstieg in die zunehmend komplexeren Ablationsverfahren zu gewähren.

Vorhofflimmern ist und wird auch zukünftig wichtiger Bestandteil der kardiologischen Praxis bleiben. Wir hoffen, Ihnen mit diesem Themenschwerpunkt neue und wichtige Konzepte zur Behandlung des Vorhofflimmerns näherzubringen.

\section{Literatur}

1. Breithardt G, Dobrev D, Doll N, et al. The German Competence Network on Atrial Fibrillation (AFNET). Herz 2008; 33:548-55.

2. Antz M, Hullmann B, Neufert C, et al. Antikoagulation bei Vorhofflimmern - Update. Herz 2008;33:556-60.

3. Schmitt J, Ehrlich JR, Hohnloser SH, et al. New antiarrhythmic drugs for the treatment of atrial fibrillation. Herz 2008;33:562-7.

4. Straube F, Dorwarth U, Janko S, et al. Frequenzkontrolle versus Rhythmuskontrolle mit und ohne Herzinsuffizienz. Herz 2008;33:568-79.

5. Haïssaguerre M, Jaïs P, Shah DC, et al. Spontaneous initiation of atrial fibrillation by ectopic beats originating in the pulmonary veins. NEJM 1998;339:659-66.

6. Schmidt B, Chun KRJ, Metzner A, et al. Balloon catheters for pulmonary vein isolation. Herz 2008;33:580-4.

7. Chun KRJ, Schmidt B, Köktürk B, et al. Catheter ablation new developments in robotics. Herz 2008;33:586-9.
Herz 2008;33:547

DOI 10.1007/

s00059-008-3190-5

Korrespondenzanschrift

Prof. Dr. Karl-Heinz Kuck II. Medizinische Abteilung Asklepios Klinik St. Georg Lohmühlenstraße 5 20099 Hamburg Telefon (+49/40) 181885-2305, Fax -4444 E-Mail:k.kuck@ asklepios.com 